\title{
Throughput maximization in distributed cognitive radio wireless mesh networks using lagrangian duality theory
}

\author{
G.Suganya ${ }^{1}$, Mr.N.Khadar basha ${ }^{2}$, \\ ${ }^{I}$ (M.E, Communication systems, Dhanalakshmi srinivasan engineering college, Perambalur, India.) \\ ${ }^{2}$ (Assistant professor of ECE department, Dhanalakshmi srinivasan engineering college, Perambalur, India.)
}

\begin{abstract}
A cognitive radio network improves the spectrum utilization. In this paper, we present a distributed algorithm for scheduling and spectrum allocation, they maximize the networks throughput. During each time slot, the scheduling and spectrum allocation problems selecting a subset of links to be activated, and allocate the available resources to these links. This problem is considered as an aggregate utility maximization problem. The queuing analysis used to characterize the throughput and delay performance of the network and throughput is maximized by Lagrangian duality theory. The problems are divided into a set of sub problems that can be solved locally by using dual decomposition framework, \& it allows us to develop distributed algorithm.
\end{abstract}

Keywords: cognitive radios, distributed algorithm, lagrangian duality theory, Resource allocation.

\section{INTRODUCTION}

Cognitive radio is a radio that is able to sense the spectral environment over wide frequency band \& exploit this information to opportunistically provide wireless links that best meet the user communication requirements. In CR networks two users are available. They are primary user \& secondary user. It allows SUs to utilize spectrum holes left by the primary user without causing any interference to PUs. One of the biggest challenges in cognitive radio networks is spectrum sharing, which defines the set of rules and strategies that regulate the behaviour of SUs regarding spectrum mobility, allocation, and access. The spectrum sharing architectures are centralized and distributed. In centralized network, a centralized entity controls both spectrum allocation and access. In a distributed architecture, each SU is responsible for the channel allocation and access decisions. The SU may make its decisions based on its local observation of the network and spectrum status or by cooperating with other SUs to have a more global observation. The cognitive radio mesh networks are used to reduce congestion in traditional WMNs by searching for available channels in the primary band. In [4], three different frequency assignment problems are considered. They are common broadcast frequencies, non interfering frequencies for simultaneous transmissions, and frequencies for direct source-destination communications. However, these algorithms only guarantee non-interfering direct communication between pairs of nodes. In [7], bandwidth allocation problem is considered, in which max-min fairness models and heuristic algorithms are used. The algorithm requires global information about the network to be collected at a central point \& it considered only bandwidth allocation problem and not consider any performance measures.

This paper studies the resource allocation problem in cognitive radio mesh networks. The objective is to maximize the end to end throughput of the different traffic streams in the network. The problem is considered as an aggregate utility maximization problem, and they provides fairness guarantees among different streams. The queuing model enables us to characterize the throughput as well as the delay at each node in the network. The non-linear integer programming (NIP) problem is considered as utility maximization problem. By changing the integer valued into decision variables. So these cause some problem. These can be avoided by dual decomposition of the problem.

\section{SYSTEM MODEL}

The cognitive radio based wireless mesh network model used is shown in Fig. 1. The cognitive mesh network consists of $\mathrm{M}$ nodes that opportunistically share the spectrum resources with a primary network composed of $\mathrm{N}$ transmitter-receiver pairs. Each primary user operates its own channel that does not overlap with other users' channels. Therefore, there will be $\mathrm{N}$ non-overlapping channels. Furthermore, all primary channels have the same bandwidth. We assume that the primary network follows a time-slotted transmission structure. So they can start only at the beginning of a time slot. Based on the spectrum sensing techniques, the local channel availability can be detected. 


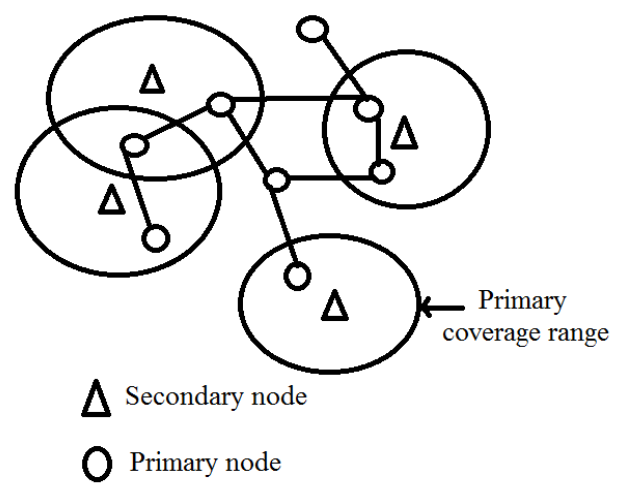

Fig. 1.Network model.

The network uses hybrid TDMA for channel access. In each time slot, a node selects one of the available frequency channels to transmit over. The cognitive network adjusts the boundaries of its time slots and matches it with primary network.

A cognitive mesh node senses its assigned channel cat the beginning of each time slot $t$. If the channel is idle, the node transmits the packet at the head of its queue to the next node along the route to its destination. otherwise it remains silent and keeps sensing the channel in subsequent time slots. For simplicity, we will assume that cognitive nodes have access to perfect spectrum sensing information. The case of imperfect sensing, where SUs can sometimes make false detections, can be easily incorporated into the problem formulation similar to the model. After the successful transmission, the receiving node sends ACK packet to source node. The cognitive mesh network is modelled as a directed graph $\mathrm{G}(\mathrm{V}, \mathrm{E})$, where $\mathrm{V}$ is vertex and $\mathrm{E}$ is edge. Each vertex $v \in V$ corresponds to a cognitive mesh node. An edge $\mathrm{e} \in \mathrm{E}$ exists between nodes $\mathrm{u}$ and $\mathrm{v}$ if there exists a channel $\mathrm{c}$ and the nodes are within transmission range of each other.

\subsection{Queuing Model}

Rayleigh flat fading channel with additive white Gaussian noise is taken as the wireless channel between a node and its destination. Each node has an infinite buffer for storing packets of fixed length. The finite buffers case could also be accommodated into our model with slight modifications to the optimization problem.

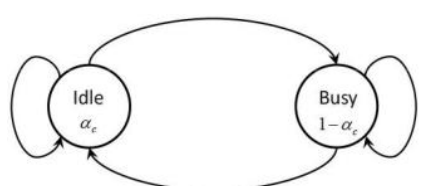

Fig.2. Markov chain model.

The primary channels are modelled using a two state Markov chain as shown in Fig. 2. If the channel c is idle the Markov chain denotes off state. Otherwise they denotes on state. Node $u$ as the source and they have data stream $\mathrm{f}$. The packet arrivals at the source are modelled as a stationary Bernoulli process.

\section{QUEUING ANALYSIS}

In queuing analysis, first the arrival and service is calculated. In this we are consider time and channel as resource element.

\section{Arrival rate}

The average arrival and service rates can be calculated using decision variables. The decision variables:

- $\quad y_{f, e}^{t}, c=1$ if the resource element is allocated to data stream $f$; otherwise $y_{f}^{t, c}, e=0$.

The data stream $f$ enters the queue of node $v$ in a given TDMA frame if:

1. Time and channel allocated to one of v's incoming link,

2. The primary user channel is idle during that time slot

3. The previous cognitive node in the route has at least one packet in its queue to transmit to node $\mathrm{v}$. The joint probability will be:

$$
\mathrm{a}_{\mathrm{v}}^{\mathrm{f}}=\sum_{\mathrm{e} \in \mathrm{E}_{\mathrm{v}}^{\text {in }}} \sum_{\mathrm{c}=1}^{\mathrm{N}} \mathrm{y}_{\mathrm{f}, \mathrm{e}}^{\mathrm{t}, \mathrm{c}} \frac{\lambda_{\mathrm{e}(\mathrm{S})}^{\mathrm{f}}}{\mu_{\mathrm{v}}^{\mathrm{f}}}\left[\mathrm{I}_{\mathrm{e}}^{\mathrm{c}} \alpha_{\mathrm{c}}+\left(1-\mathrm{I}_{\mathrm{e}}^{\mathrm{c}}\right)\right]\left(1-\mathrm{p}_{\mathrm{e}}^{\text {out }}\right)
$$

Where $\mathrm{E}_{\mathrm{v}}^{\mathrm{in}}$ is the set of incoming link to node $\mathrm{v}, \mathrm{e}(\mathrm{s})$ is the source node for link, $\lambda_{\mathrm{e}(\mathrm{s})}$ is its arrival rate 
and $\mu_{\mathrm{e}(\mathrm{s})}^{\mathrm{f}}$ its service rate. The average packet arrival probability will be:

$$
\lambda_{v}^{\mathrm{f}}=1 / \mathrm{T} \sum_{\mathrm{t}=1}^{\mathrm{T}} \mathrm{a}_{\mathrm{v}}^{\mathrm{f}, \mathrm{t}}
$$

which is interpreted as the probability that a packet from data stream $\mathrm{f}$ arrives at node $\mathrm{v}$ in any time slot. Given this definition, packet arrivals can be seen as Bernoulli trials at each time slot with success probability $\lambda_{\mathrm{v}}^{\mathrm{f}}$. Therefore, the packet arrival process can be approximated as a Bernoulli process with average arrival rate $\lambda_{\mathrm{v}}^{\mathrm{f}}$.

\section{Service rate}

To calculate the average service rate, we start by identifying the events necessary for a successful packet transmission. This will take place if in a given time slot $\mathrm{t}$

1. Channel and time assigned to one of v's outgoing links,

2. The primary user channel is either idle during that time slot.

The joint probability will be:

$$
\mathrm{s}_{\mathrm{v}}^{\mathrm{f}}=\sum_{\mathrm{e} \in \mathrm{E}_{\mathrm{v}}^{\text {in }}} \sum_{\mathrm{c}=1}^{\mathrm{N}} \mathrm{y}_{\mathrm{f}, \mathrm{e}}^{\mathrm{t}, \mathrm{c}}\left[\mathrm{I}_{\mathrm{e}}^{\mathrm{c}} \alpha_{\mathrm{c}}+\left(1-\mathrm{I}_{\mathrm{e}}^{\mathrm{c}}\right)\right]\left(1-\mathrm{p}_{\mathrm{e}}^{\text {out }}\right)
$$

Where $\mathrm{E}_{\mathrm{v}}^{\text {out }}$ is the set of outgoing edges to node $\mathrm{v}$. The average service probability will be:

$$
\mu_{v}^{\mathrm{f}}=1 / \mathrm{T} \sum_{\mathrm{t}=1}^{\mathrm{T}} \mathbf{s}_{\mathrm{v}}^{\mathrm{f}, \mathrm{t}}
$$

which is the probability that a packet from data stream $\mathrm{f}$ leaves the queue of node $\mathrm{v}$ in any time slot. Similar to the arrival events, the service events can be seen as Bernoulli trials at each time slot with success probability. Therefore, the packet service process can be approximated as a Bernoulli process with average service rate.

\section{FORMULATION OF OPTIMIZATION PROBLEM}

First, we consider the utility function. The utility function can be maximized using optimization problem then they find resource allocation solution. This can be formulated as follows:

$$
\begin{aligned}
& \max _{\bar{y}}^{\max } \sum_{\mathrm{f} \in \mathrm{F}} \mathrm{U}_{\mathrm{f}}\left(\min _{\mathrm{v} \in \mathrm{v}_{\mathrm{f}}} \mu_{\mathrm{v}}^{\mathrm{f}}\right) \\
& \mathrm{y}_{\mathrm{f}, \mathrm{e}}^{\mathrm{t}, \mathrm{c}} \in\{0,1\}
\end{aligned}
$$

With respect to Constraints are,

The objective function is to maximize the aggregate utility function for all the traffic streams, where $\mathrm{F}$ is set of all traffic streams, and Vf is the set of nodes along the end-to-end path for traffic stream $\mathrm{f}$. If the available radios can only access a single channel at any given time slot means they can be modified to accommodate multi-channel radios, as well as different capabilities for different nodes. The interfering links are allocated distinct resource elements, which avoids interference between mesh nodes during packet transmissions. The function provides guarantees to the stability of all the queues in the network. Finally, constraint (6) ensures that the decision variable can only take a value of 0 or 1.

Presence of the min term inside the utility function makes the problem difficult to solve. To simplify this, we transform the min term into a set of linear inequality constraints. This transformation simplifies the objective function and allows us to use the duality theory to find a decentralized solution. Let $\overline{\mathbf{z}}=\left\{\mathrm{z}_{\mathrm{f}}, \mathrm{f} \in \mathrm{F}\right\}$ be the vector of such decision variables.

$$
\max _{\mathrm{y} z}^{-} \sum_{\mathrm{f} \in \mathrm{F}} \mathrm{U}_{\mathrm{f}}\left(\mathrm{z}_{\mathrm{f}}\right)
$$

Then the equivalent optimization problem can be written as,

$$
\mathrm{z}_{\mathrm{f}}<\mu_{\mathrm{v}}^{\mathrm{f}}, \forall \mathrm{f} \in \mathrm{F}, \mathrm{v} \in \mathrm{V}_{\mathrm{f}}
$$

The maximization is now over the two sets of decision variables, $\mathrm{y}_{\mathrm{f}, \mathrm{e}}^{\mathrm{t,c}}$ and $\mathrm{zf}$. Each min term in the original optimization problem is now replaced by $|\mathrm{Vf}|$ linear inequality constraints.

\subsection{Real value to binary value conversion}

The high complexity of the nonlinear integer programming problem can be reduced by using the real value to binary value conversion and allow the decision variables to take real values in the interval $[0,1]$.

$$
\mathrm{y}_{\mathrm{f}, \mathrm{e}}^{\mathrm{t}, \mathrm{c}} \in[0,1] \text {, }
$$

This conversion transforms the nonlinear integer program into a convex optimization problem with real valued variables. However, the resulting optimal solution for the relaxed problem is not guaranteed to be optimal for the original integer-valued problem. 


\section{Convex function}

A real-valued function $\mathrm{f}(\mathrm{x})$ defined on an interval is called convex or convex downward or concave upward if the line segment between any two points on the graph of the function lies above the graph, in a Euclidean space or vector space of at least two dimensions. Equivalently, a function is convex if its epigraph the set of points on or above the graph of the function) is a convex set.

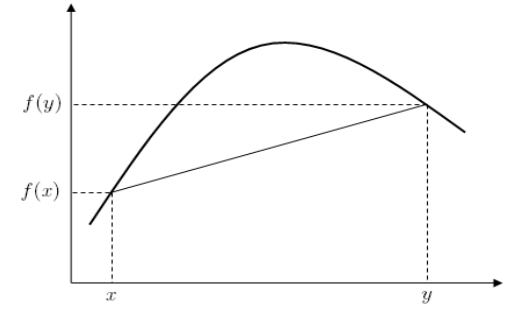

The algorithm starts by defining the set $\mathrm{X}$ of all resource elements that are assigned to any of the network links. Then the highest assignment probability is found and the corresponding decision variable is set to $\mathrm{y}_{\mathrm{f}, \mathrm{e}}^{\mathrm{t}, \mathrm{c}}=1$. Given the constraints in IV a set $\mathrm{Q}$ of all the resource elements with assignments that conflict with the above assignment is defined. All the conflicting assignments are then released, and the set $\mathrm{X}$ is updated by removing the element $\left(\mathrm{f}^{*}, \mathrm{e}^{*}, \mathrm{t}^{*}, \mathrm{c}^{*}\right)$ and all elements in $\mathrm{Q}$ from $\mathrm{X}$. These steps are repeated till all the elements are removed from the set $\mathrm{X}$.

\section{DISTRIBUTED CRN NETWORK}

In distributed network calculations are done locally at each node, or at local central points or cluster heads is desirable. In this section, we propose a decomposition of the original problem into smaller subproblems that can be efficiently solved in a distributed fashion.

\subsection{LAGRANGIAN DUALITY THEORY}

The distributed solution approach is based on dual decomposition. The first step is to define the Lagrangian function for the optimization problem in (11) as follows

$$
\begin{aligned}
\mathrm{L}= & \sum_{\mathrm{f} \in \mathrm{F}} \mu_{\mathrm{f}}\left(\mathrm{z}_{\mathrm{f}}\right)-\sum_{\mathrm{f} \in \mathrm{F}} \sum_{\mathrm{v} \in \mathrm{V}_{\mathrm{f}}} \mathrm{p}_{\mathrm{f}, \mathrm{v}}^{1}\left(\mathrm{z}_{\mathrm{f}}-\mu_{\mathrm{f}}\right)-\sum_{\mathrm{v} \in \mathrm{V}} \sum_{\mathrm{t}=1}^{\mathrm{T}} \mathrm{p}_{\mathrm{f}, \mathrm{v}}^{2}\left[\sum_{\mathrm{f} \in \mathrm{F}} \sum_{\mathrm{e} \in \mathrm{E}_{\mathrm{v}}^{\text {out }}} \sum_{\mathrm{c}=1}^{\mathrm{N}} \mathrm{y}_{\mathrm{f}, \mathrm{e}}^{\mathrm{t}, \mathrm{e}}-1\right]- \\
& \sum_{\mathrm{v} \in \mathrm{V}} \sum_{\mathrm{t}=1}^{\mathrm{T}} \mathrm{p}_{\mathrm{f}, \mathrm{v}}^{3}\left[\sum_{\mathrm{f} \in \mathrm{F}} \sum_{\mathrm{c}=1}\left(\sum_{\mathrm{e} \in \mathrm{E}_{\mathrm{v}}^{\text {in }}} \mathrm{y}_{\mathrm{f}, \mathrm{e}}^{\mathrm{t}, \mathrm{c}}+\sum_{\mathrm{e} \in \mathrm{E}_{\mathrm{v}}^{\mathrm{in}}} \mathrm{y}_{\mathrm{f}, \mathrm{e}}^{\mathrm{t}, \mathrm{c}}\right)-1\right]-\quad \sum_{\mathrm{e} \in \mathrm{E}} \sum_{\mathrm{v} \in \mathrm{V}} \sum_{\mathrm{t}=1}^{\mathrm{T}} \mathrm{p}_{\mathrm{e}, \mathrm{t}, \mathrm{c}}^{4}\left[\sum_{\mathrm{f} \in \mathrm{F}} \sum_{\mathrm{e} \in \mathrm{s}_{\mathrm{e}}} \mathrm{y}_{\mathrm{f}, \mathrm{e}}^{\mathrm{t}, \mathrm{c}}-\right. \\
& 1 \mathrm{f} \in \mathrm{Fv} \in V f p f, \mathrm{v} 5 \lambda \mathrm{ff}-\mu \mathrm{vf}
\end{aligned}
$$

wherep ${ }_{\mathrm{f}, \mathrm{v}}^{1}, \mathrm{p}_{\mathrm{v}, \mathrm{t}}^{2}, \mathrm{p}_{\mathrm{v}, \mathrm{t}}^{3}, \mathrm{p}_{\mathrm{e}, \mathrm{t}, \mathrm{c}}^{4}$ and $\mathrm{p}_{\mathrm{f}, \mathrm{v}}^{5}$ are the Lagrange multipliers. Where Ef is the set of edges forming the endto-end path for traffic stream $\mathrm{f}$.

$$
\begin{aligned}
& \mathrm{L}= \\
& \quad \sum_{\mathrm{f} \in \mathrm{F}}\left[\mathrm{u}_{\mathrm{f}}\left(\mathrm{z}_{\mathrm{f}}\right)-\sum_{\mathrm{v} \in \mathrm{V}_{\mathrm{f}}} \mathrm{p}_{\mathrm{f}, \mathrm{v}}^{1}\left(\mathrm{z}_{\mathrm{f}}-\mu_{\mathrm{f}}\right)-\sum_{\mathrm{v} \in \mathrm{V}} \sum_{\mathrm{t}=1}^{\mathrm{T}} \sum_{\mathrm{e} \in \mathrm{E}_{\mathrm{v}}^{\text {out }}} \sum_{\mathrm{c}=1}^{\mathrm{N}} \mathrm{p}_{\mathrm{f}, \mathrm{v}}^{2} \mathrm{y}_{\mathrm{f}, \mathrm{e}}^{\mathrm{t}, \mathrm{c}}-1-\right. \\
& \mathrm{v} \in \mathrm{Vt}=1 \mathrm{Tpf}, \mathrm{v} 3 \mathrm{c}=1 \mathrm{e} \in \text { Evinyf,et,c }+\mathrm{e} \in \text { Evinyf,et,c- }-\mathrm{v} \in \mathrm{Vft}=1 \mathrm{Tpf}, \mathrm{v} 3-\mathrm{e} \in \mathrm{Ev} \in \mathrm{Vt}=1 \mathrm{Tpf}, \mathrm{v} 4 \mathrm{e} \in \text { seyf,et,c- } \\
& \mathrm{e} \in \mathrm{Ev} \in \mathrm{Vt}=1 \mathrm{Tpf}, \mathrm{v} 4-\mathrm{f} \in \mathrm{Fv} \in \mathrm{Vfpf}, \mathrm{v} 5 \lambda \mathrm{vf}-\mu \mathrm{vf}
\end{aligned}
$$

From (15) it is concluded that this Lagrangian can be divided into $|\mathrm{F}|$ separate sub-problems, one for each of the traffic streams in the network. Each sub-problem for stream $\mathrm{f}$ can be solved locally if the values of the Lagrange multipliers $\mathrm{p}_{\mathrm{f}, \mathrm{v}}^{1}, \mathrm{p}_{\mathrm{v}, \mathrm{t}}^{2}, \mathrm{p}_{\mathrm{v}, \mathrm{t}}^{3}, \mathrm{p}_{\mathrm{e}, \mathrm{t}, \mathrm{c}}^{4}$, and $\mathrm{p}_{\mathrm{f}, \mathrm{v}}^{5}$ at each node or link taking part in the routing path for stream $f$ are known.

The dual problem can then be written as:

$$
\underset{--}{\max \min L}
$$

\subsection{Conversion of convex function into concave function}

The non-differentiable dual objective function appear means, its gradient may not always exist. This is because in general, differentiability of the dual requires a unique primal optimizer whereas in our case, the optimal values of the variables can be non-unique. Therefore, the well-known gradient-based algorithms do not apply in this case. The reason behind the non-differentiability of the dual objective function is the lack of strict concavity of the primal objective function.

\section{Concave function}

A concave function is the negative of a convex function. A concave function is also called concave downwards, concave down, convex upwards, convex cap or upper convex. A real-valued function $f$ on an interval is said to be concave if, for any $\mathrm{x}$ and $\mathrm{y}$ in the interval and for any $\mathrm{t}$ in $[0,1]$. 
The solution approach we present here is based on the proximal minimization algorithm. To make the primal objective function strictly concave, a strictly concave term is added for each of the variables $\mathrm{y}_{\mathrm{f}, \mathrm{e}}^{\mathrm{t}, \mathrm{c}}$ therefore, making the dual function differentiable with respect to all decision variables. For each variabley $\mathrm{f}_{\mathrm{f}, \mathrm{e}}^{\mathrm{t}, \mathrm{c}}$, we introduce an additional variable $\mathrm{x}_{\mathrm{f}, \mathrm{e}}^{\mathrm{t}, \mathrm{c}}$ anddefinex as the vector containing these variables.

The approximate primal objective function is now written as:

$$
\stackrel{\max }{\mathrm{y} z \mathrm{x}} \sum_{\mathrm{f} \in \mathrm{F}} \mathrm{U}_{\mathrm{f}}\left(\mathrm{z}_{\mathrm{f}}\right)-\sum_{\mathrm{f} \in \mathrm{F}} \sum_{\mathrm{e} \in \mathrm{E}_{\mathrm{v}}^{\text {out }}} \sum_{\mathrm{t}=1}^{\mathrm{T}} \sum_{\mathrm{c}=1}^{\mathrm{N}} \frac{1}{2 \mathrm{~K}}\left(\mathrm{y}_{\mathrm{f}, \mathrm{e}}^{\mathrm{t}, \mathrm{c}}-\mathrm{x}_{\mathrm{f}, \mathrm{e}}^{\mathrm{t}, \mathrm{c}}\right)
$$

Subject to constraints in IV. Since the primal objective function is now strictly concave, the dual is differentiable, and the gradient of $\mathrm{L}^{1}$ with respect to the different Lagrange multipliers. Applying the gradient projection method, the Lagrange multipliers are calculated iteratively. Then the decision variables are determined.

\subsection{Algorithm implementation in distributed network}

The proximal approximation algorithm can be implemented in a real network in the following way. At the ith iteration, any node $\mathrm{v}$ updates the Lagrange multipliers associated with itself and with all outgoing links. Then, using the updated multipliers, node $\mathrm{v}$ calculates the resources allocated to each of its outgoing links.

Each node will need to identify all the nodes are within its interference range by using hello protocol. At each iteration, information exchange between nodes can be classified into 4 categories as follows;

1) Each node broadcasts its resource allocation solutions to all nodes within interference range.

2) The last node in any given route forwards its $p_{f, v}^{1}$ value to the next node on the backward path towards the source node. Subsequent nodes on that backward path add their own $\mathrm{p}_{\mathrm{f}, \mathrm{v}}$ value to the value received from preceding nodes and then forward the sum to the next node towards the source.

3) Each node forwards its $\mathrm{p}_{\mathrm{v}, \mathrm{t}}^{3}$ value to the next node along the backward path towards the source node. Transmission of $\mathrm{p}_{\mathrm{v}, \mathrm{t}}^{3}$ and accumulated $\mathrm{p}_{\mathrm{f}}^{1}$, values can be combined into a single packet transmission at each node, since both values are propagated in the backward path.

4) Once the source calculates $z_{f}$, this value is transmitted propagated along the forward path from the source towards the destination.

\section{RESULTS}

Performance of the proposed resource allocation algorithm is compared with uniform resource and max-min bandwidth allocation (MMBA) algorithm. In uniform resource allocation the resources are uniformly distributed. In fig 4 it is clear from the figures that our proposed scheme is able to support the secondary nodes traffic at lower primary channels idle probability compared to the two other schemes.

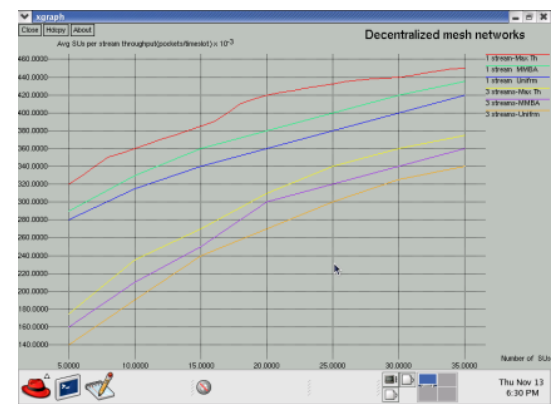

Fig. 3(a) Number of secondary nodes vs. average secondary throughput

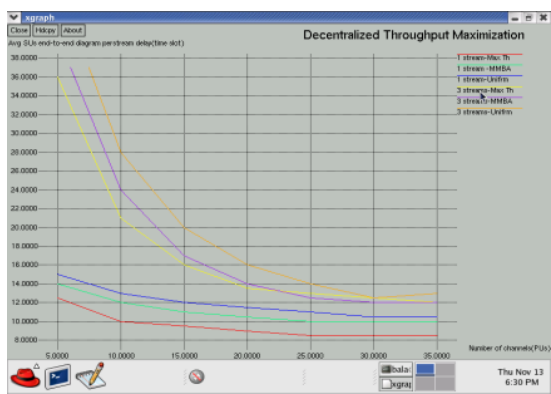

Fig. 3(b) Number of secondary nodes vs. secondary end-to-end delay 


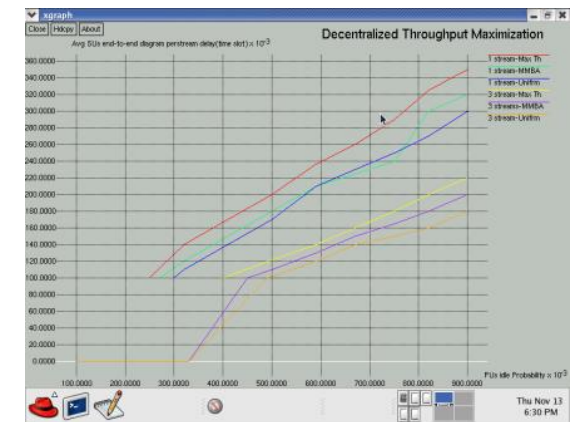

Fig. 4(a) PUs idle probability vs. secondary throughput

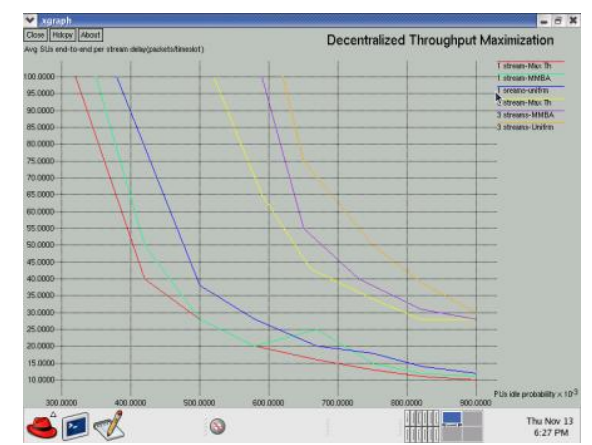

Fig. 4(b) Primary users idle probability vs. average secondary end-to-end delay.

In Fig. 5(a), it is noted that the proposed resource allocation algorithm outperforms than both the MMBA and the uniform allocation schemes, in the case of 1 traffic stream, and the case of 3 traffic streams. Moreover, to accommodate the 3 traffic streams, the MMBA and uniform allocation schemes require 5 channels, while the proposed schemes requires only 4.

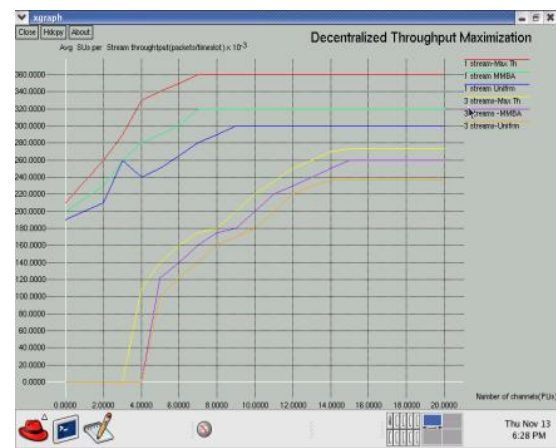

Fig. 5(a) Number of primary channels Vs average secondary throughput.

The end-to-end delay performance as a function of the number of primary channels in Fig. 5(b).For instance, there is a $20 \%$ decrease in the average delay for the proposed algorithm compared to uniform allocation, and $12 \%$ decrease compared to MMBA.

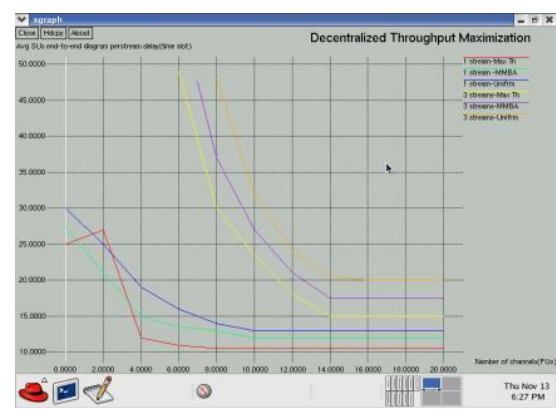

Fig. 5(b) Number of primary channels vs. SUs end-to-end delay 


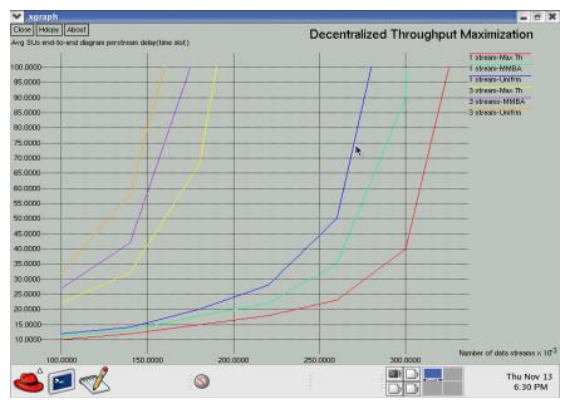

Fig.6. Secondary arrival rate on secondary end-to-end delay

For instance, with 5 channels our scheme is able to support all the 5 traffic streams, while the MMBA scheme can support 4 streams and the uniform scheme supports only 3 streams in fig 7 .

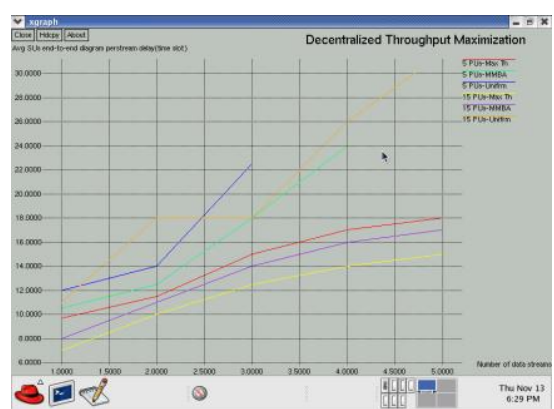

Fig.7. Number of traffic streams vs. secondary end-to-end delay.

The effect of the delay constraint parameter d on the network's performance is depicted in Fig.8 the network has 15 secondary nodes. It is noted that the achievable throughput by the secondary nodes is almost unaffected by the delay constraint.

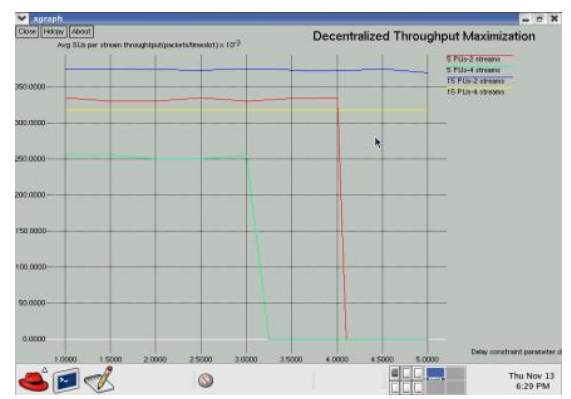

Fig.8. Delay constraint parameter vs. avg. secondary throughput.

\section{CONCLUSION}

Utility maximization problem is considered as throughput maximization problem in cognitive radio based WMNs. The utility function provides a degree of fairness among different streams. The resource allocation problem in centralized network was decomposed into a set of sub-problems. The resource allocation problem was decomposed into a set of sub-problems. Then the problems are locally solved. The proposed algorithm improves the throughput of the network. Results demonstrate the efficiency of the proposed decentralized solution scheme, adapt varies network loads. Performance gains of the proposed method are compared with uniform and max-min band-width allocation. The proposed method accommodates more traffic streams and they increases throughput and decreases delay compared to other algorithm.

\section{REFERENCES}

[1] S. A. Zekavat and X. Li, "User-central wireless system: Ultimate dynamic channel allocation," in Proc. IEEE DySPAN, Baltimore, MD, USA, Nov. 2005, pp. 82-87.

[2] J. Zhao, H. Zheng, and G.-H. Yang, "Distributed coordination in dynamic spectrum allocation networks," in Proc. IEEE DySPAN, Baltimore, MD, USA, Nov. 2005, pp. 259-268.

[3] L. Cao and H. Zheng, "Distributed spectrum allocation via local bargaining," in Proc. IEEE Conf. SECON , Santa Clara, CA, 
USA, Sep.2005, pp. 475-486.

[4] M. E. Steenstrup, "Opportunistic use of radio-frequency spectrum: A network perspective,"in Proc. IEEE DySPAN, Baltimore, MD, USA,Nov. 2005, pp. 638-641.

[5] T.Chen, H.Zhang, G.M.Maggio, and I.Chlamtac, "Topology management in CogMesh: A cluster-based cognitive radio mesh network," in Proc. IEEE ICC, Glasgow, U.K., Jun. 2007, pp. 6516-6521.

[6] M. Sharma, A. Sahoo, and K. D. Nayak, "Channel selection under interference temperature model in multi-hop cognitive mesh networks," in Proc. IEEE DySPAN, Dublin, Ireland, Apr. 2007,pp. 133-136.

[7] J. Tang, R. Hincapie, G. Xue, W. Zhang, and R. Bustamante, "Fair bandwidth allocation in wireless mesh networks with cognitive radios,"IEEE Trans. Veh. Technol., vol. 59, no. 3, pp. 1487-1496, Mar. 2010.

[8] C. Xin, B. Xie, and C.-C. Shen, "A novel layered graph model for topology formation and routing in dynamic spectrum access networks," in Proc. IEEE DySPAN, Baltimore, MD, USA, Nov. 2005,308-317.

[9] A. El-Sherif, A. Mohamed, and Y. C. Hu, "Joint routing and resource allocation for delay sensitive traffic in cognitive mesh networks,” in Proc. IEEE Globecom Workshop RACCN, Houston, TX, USA, Dec.2011, pp. 947-952.

[10] F. P. Kelly, A.Maulloo, and D.Tan, "Rate control for communica-tion networks: Shadow prices, proportional fairness and stability,” J. Operat. Res. Society, vol. 49, no. 3, pp. 237- 252, 1998.pp.947-952

[11] D. Cabric, S. M. Mishra, and R. W. Brodersen, "Implementation issues in spectrum ensing for cognitive radio," in Proc. Asilomar Conf. Signals, System, Computer, Pacific Grove, CA, USA, 2004,pp.772-776.

[12] S.Enserink and D. Cochran, "A cyclostationary feature detector," in Proc. 28th Asilomar Conf. Signals, Systems, and Computers, Pacific Grove, CA, USA, Oct. 1994, pp. 806-810.

[13] A. El-Sherif, A. K. Sadek, and K. J. R. Liu, "Opportunistic multiple access for cognitive radio networks," IEEE J. Select. Areas Commun., vol. 29, no. 4, pp. 704-715, Apr.2011.

[14] R. A. Tannious and A. Nosratinia, "Cognitive radio protocols based on exploiting hybrid ARQ retransmissions," IEEE Trans.Wireless Commun., vol. 9, no. 9, pp. 2833-2841, Sep. 2010.

[15] M. Levorato, U.Mitra, and M. Zorzi, “ Cognitive interference management in retransmission-based wireless networks," IEEE Trans .Inf. Theory, vol. 58, no. 5, pp. 3023-3046, May 2012.

[16] A. El-Sherif and K. J. R. Liu, "Joint design of spectrum sensing and channel access in cognitive radio networks, " IEEE Trans.Wireless Commun., vol. 10, no. 6, pp.1743-1753, Jun. 2011. 